\title{
MOBILITY MANAGEMENT IN HETEROGENEOUS WIRELESS NETWORKS
}

\author{
G. Zayaraz ${ }^{1}$, J. Kanchana Devi ${ }^{2}$, V. Vijayalakshmi ${ }^{3}$, V. Hemamalini ${ }^{4}$ \\ ${ }^{1}$ Professor, Department of CSE, Pondicherry Engineering College, Puducherry \\ ${ }^{2}$ M.Tech Student, Department of CSE, Pondicherry Engineering College, Puducherry \\ ${ }^{3}$ Assistant Professor, Department of ECE, Pondicherry Engineering College, Puducherry \\ ${ }^{4}$ Research scholar, Department of CSE, Pondicherry Engineering College, Puducherry
}

\begin{abstract}
Ever increasing demands of users and rapid development of telecommunication technologies have led to the evolution of $4 G$ heterogeneous wireless network. Further, $4 G$ with heterogeneous network environment will provide features such as seamless internet connectivity between different wireless networks, "Always Best Connected" service and "Anywhere Anytime Service". Due to the different characteristics of heterogeneous wireless networks such as network coverage, bandwidth, data rate etc designing an efficient vertical handoff is the most challenging issue. To provide a seamless connectivity to the mobile users, there is a strong demand for the integration of different wireless access networks. The Worldwide Interoperability for Microwave Access (IEEE 802.16 WIMAX) networks has the capability to provide global network coverage with high data rate, but their deployment cost is high. The Wireless Local Area Network (IEEE 802.11 WLAN) has the capability to provide high data rate at a very low cost but their services are limited to a very small geographical region. By integrating these two challenging technologies WIMAX and WLAN several benefits can be achieved, i.e. increased network coverage, load balancing and better Quality of Service (QoS). Therefore, the integration of WLAN and WIMAX provides seamless internet connectivity and high data rate at low cost to the wireless mobile users. In this paper different integration mechanisms of WIMAX and WLAN are investigated. To be more precise, an internetworking mechanism for the integration of WLAN and WIMAX based on two different types of coupling, i.e loose coupling and tight coupling are implemented. Also an efficient vertical handoff based on IEEE 802.21 Media Independent Handoff approach is implemented on different interworking architecture of WLAN and WIMAX. The simulated results shows that the tight coupling is better than the loose coupling for the applications implemented using Opnet. And also it is found that an efficient handover can be achieved when handover is done based on signal strength and bandwidth.
\end{abstract}

Keywords: Heterogeneous wireless network, Loose coupling, Tight Coupling, Mobile IP, MIH, Point of Attachment, Received Signal Strength

\section{INTRODUCTION}

Heterogeneous wireless network refers to interconnection of different wireless network with different underlying radio access technologies. $4 \mathrm{G}$ network will provide features such as "Always Best Connected" which means mobile users being connected to the best available networks. For instance if a user gets Internet Connectivity through broadband connectivity at home and if the user is on move, he should be ensured of seamless connectivity till he reaches destination.

There are several benefits to a Heterogeneous Wireless Network as opposed to a traditional Homogeneous wireless network including increased reliability, improved spectrum efficiency, improved quality of service and increased network coverage. Reliability is also improved because when one particular Radio Access Technology within the Heterogeneous Wireless Network fails, it may still be possible to maintain a connection by falling back to another The remainder of the paper is organized as follows: Section 2 discusses about Related Work. In Section 3, an overview of WLAN and WIMAX network is discussed. Section 4 discusses about Mobility Management and Section 5 presents proposed vertical handover between WLAN and WIMAX. Section 6 evaluates the performance of vertical handoff in several important metrics and compares the results with those of the other approach. Section 7 draws concluding remarks and directions for future work.

\section{RELATED WORKS}

Stenio[1] gave a widespread review on different mobility management architectures for seamless vertical handover of mobile users in different wireless access networks. He also proposed Context Aware Mobility Management architecture (CAMMS) that does handover based on the improvement of QOS. The studies by Farah [2] dealt with media independent services where link layer information is provided to the upper layers during pre-handover stage.

Son [3] proposed an enhanced vertical handover with $\mathrm{MIH}$ services and reduced the handoff delay by introducing ADAD mechanism. In [4] Abhishek implemented horizontal and vertical handover for WLAN and WIMAX. Here master IP address is assigned by the server and all data will be 
routed from the server to MIP. Safdar[5] implemented two different interworking techniques for the integration of WLAN and UMTS networks. The intrinsic technical differences such as of protocols, network coverage areas, data rate, handoff mechanism etc between two promising technologies brings a lot of challenges during integration. Two different tight coupling schemes are used for the integration of the WLAN and UMTS, namely SGSNWLAN and GGSN-WLAN integration. The performance of the GGSN-WLAN interworking is far better than that of SGSN-WLAN internetworking scenario. This is because the WLAN access point needs to have some additional capabilities to process the messages that comes from UMTS network and hence more processing power is required. Whereas in case of GGSN-WLAN integration, a simple WLAN access point is required and hence additional task is not required for communication. In [6] Khan proposed an architecture where mobile device has multiple transceivers and handover is done using one interface and service gets continued using other interface.

Jong [7] proposed Simple Mobility Management Protocol that supports seamless IP handover. It separates location management entity of MIP and promotes peer-to-peer based direct data transmission between the two Mobile Nodes. Hence global IP handover was achieved successfully, without making any changes to the existing network infrastructure. In [8] vertical handoff delay analysis was done between WLAN and UMTS using Session Initiation Protocol signalling. When analyzing the results, it shows that handoff delay getting increased when the user moves from WLAN to UMTS network when compared to the reverse movement of the mobile user. This is due to bandwidth limitation factor present in the wireless links. Therefore make-before-break handoff techniques has to be applied for SIP based mobility in heterogeneous wireless networks.

Ahmad [9] proposed a method to support MIPv6 for IPv4 backbone network; which is implemented by 6 to 4 tunneling approach. As a result, this technique helps in using MIPv6 to couple many IPv6- capable networks through central IPv4 backbones. On the other hand, it adds additional overhead of 20 byte for every packet transmission than the native MIPv6 solution. This additional overhead is due to encapsulation of IPv6 packet inside an IPv4 packet which consists of IPv4 header of 20 byte . Dheeya[10] analyzed the performance of various types of wireless networks with many applications and many types of handoff using OPNET simulator. Simulation results shows that WIMAX outperforms WLAN in all aspects.

Kenichi [11] demonstrated MIH functional services with media pre-authentication entity. Media independent preauthentication entity facilitated handover by authenticating with higher layer before L2 layer handover is made. Ammar [12] implemented Signal to Interference and Noise Ratio (SINR) based vertical handover and showed that proposed SINR based handover is far better than signal strength based handover in terms of throughput. Meriem [13]gave an overview of different types of existing vertical handover decision strategies. It has concluded that advanced evaluation functions are required to perform a better handover decision in order to provide quality of service to the user as well as for the efficient and effective use of available network resources.

\section{WLAN AND WIMAX NETWORKS}

\subsection{IEEE 802.11 WLAN}

IEEE 802.11 WLAN is a very popular wireless technology that covers small geographical region. WLAN is popularly used because deployment of network is easier and cheaper, easier maintenance and low infrastructure cost. It supports two kinds of working mode namely Adhoc mode and infrastructure mode. As shown in Fig-1. For infrastructure mode the wireless stations communicate with other wireless stations using Access Point (AP). Access Points are the ones that transmit and receive radio frequencies and are responsible for communication between wireless stations.

The set of all wireless stations that communicate with each other forms the Basic Service Set (BSS). A set of all connected BSSs forms Extended Service Set (ESS). Access Points present in an Extended Service Set are connected by means of a Distribution System (DS) and it can be both wired or wireless.

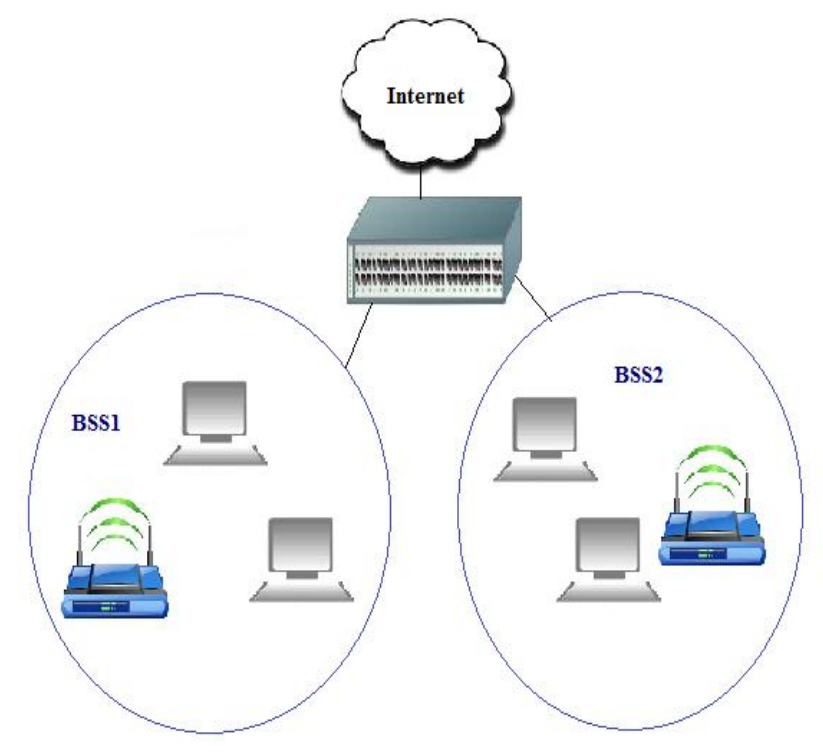

Fig-1: IEEE 802.11 WLAN Architecture

As in Fig-2. for adhoc mode, mobile device communicate with each other without the support of Access Point. In a WLAN peer-to-peer group, the group owner becomes an Access Point and all other devices in the wireless network operate as their clients. There are two methods to elect a group owner in the WLAN peer-to-peer group. In one approach, mobile node sets up a peer-to-peer group owner by itself and it can be referred to as Autonomous Group Owner. In second approach, two wireless devices compete with each other based on an intent value of the group owner. The 
wireless station that has higher intent value becomes a group owner and the other one becomes a client. The intent value depends on various factors such as remaining power of the wireless station, received signal strength of the first wireless station and whether the wireless station is already a group owner of some other group.

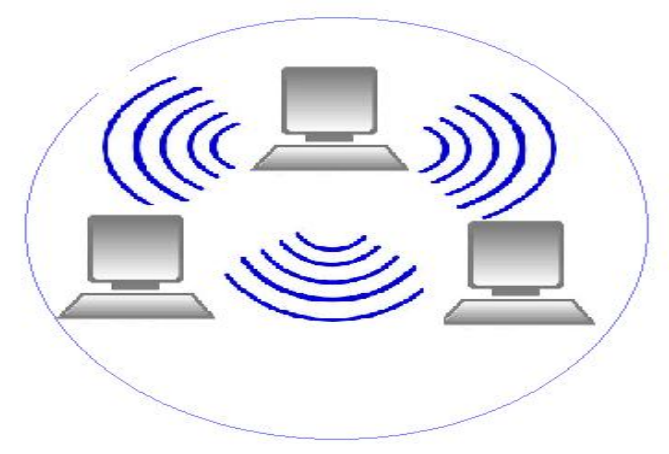

Fig-2: IEEE 802.11 Adhoc Architecture

\subsection{IEEE 802.16 WIMAX}

WIMAX is a popular wireless telecommunication technology that covers wide geographical region and works in the licensed spectrum to provide high speed wireless data transmissions. There are different kind of standards present in 802.16 and it ranges from 802.16 a to $802.16 \mathrm{~m}$. As in Fig3. WIMAX consist of several functional entities namely Mobile Station (MS)/Subscriber Station (SS), the Access Service Network (ASN) and the Connectivity Service network (CSN). The point of entry into the WIMAX network for any MS/SS is ASN. The part of the network that provides IP related services is Connectivity Service Network (CSN) and it forms the core of the network.

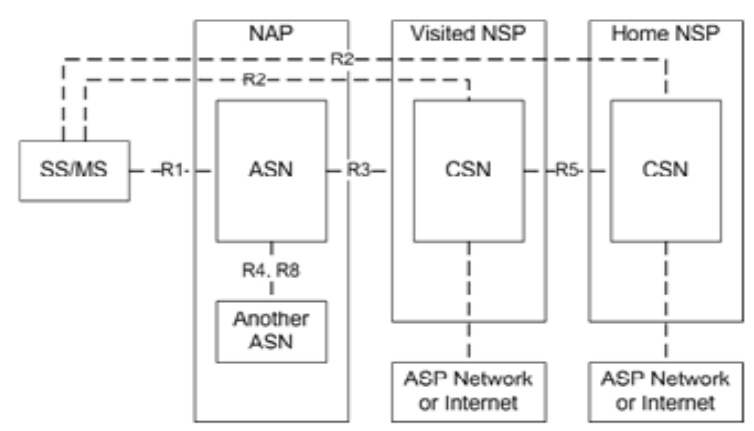

Fig-3: IEEE 802.16 WIMAX Architecture

\section{OVERVIEW OF MOBILITY MANAGEMENT}

Mobility Management consist two kind of functional entities namely location management and handoff management. Location management enables the currently serving networks to locate a mobile node's new location for delivering data packets whereas Handoff management deals with maintenance of mobile node's connection as it moves from one place to another. However Mobility management not only depends on Received Signal Strength of the candidate network but also on other parameters such as network, terminal and user. Network related parameters cover entities such as area coverage, bandwidth, latency of the network, SIR (Signal-to-Interferences Ratio), security constraints, BER (Bit Error Rate), etc. Terminal related parameters include battery power, velocity of the mobile node. User related parameters include user preferences and user policy profile.

\subsection{Location Management and Mobility}

\section{Management}

(i) Location Management: Location Management deals with tracking down the location of mobile nodes. Location management has two major functions namely location registration and call delivery. In location registration process the mobile node beacon the network periodically to update their current location in the location database and this database is kept updated from time to time. After registration is done with the current network, the call delivery process gets initiated. Based on the information that has been registered in the network database, the call delivery procedure queries the network regarding the current location of the mobile node so that a call will be delivered/ routed successfully.

(ii)Handoff management: Handoff refers to change of mobile node's point of attachment during an active communication with the end user. There are three steps in handoff process namely: Network discovery / Handoff Information phase, Handoff Decision and Handoff Execution phase.

\subsection{Types of Handover}

Depending on the number of connections maintained at the time of handoff, handoff can be classified as hard and soft. In a hard handoff the existing connection with the current network is broken, when the connection is established with the new network. To be precise, using hard handoff, a mobile node is allowed to maintain a connection with only network at any given point of time. Whereas in a soft handoff, a mobile node maintains network connection with more than one network and does not release any of the network until one of the network drops below a specific threshold value. Soft handoffs are possible in those situations where the mobile node is moving between cells of the network operating on the same frequency.

Inter-technology: When a mobile node moves from one Point of Attachment (PoA) to another of different access technologies say WIMAX, LTE, UMTS, WLAN etc., then it can be referred to as vertical handover. Since different access networks can offer significantly different datarate, bandwidth and delay, better quality of service can be achieved. 
Intra-technology: When handoff of a mobile node takes place between same network technology such as between two access points, two cellular network base station or two WIMAX base station then it can be referred to as horizontal handover. Horizontal Handover is not an important issue for the mobile users since handover takes place between networks of same access technology. Handover decision for horizontal handover is based on number of factors such as traffic across the cell, data rate, received signal strength , bandwidth etc.

\subsection{Integration Mechanism}

For effective interworking between different Radio Access Technologies a variety of methodologies is followed. They can be referred to as Loose coupling and Tight coupling.

i) Loose coupling: In loose coupling inter-networking approach, networks are deployed independently and there is no dependency between the networks. The WLAN is connected to the Internet Protocol (IP) network and maintains an indirect connection with that of the WIMAX network. So here two networks are integrated via internet as in Fig-4. The main advantage of using the loose coupling interworking is that, it facilitates independent deployment of any number of networks and allows the network service providers to utilize the resources available at another service provider. This technique requires minimal deployment changes for integration among different networks.

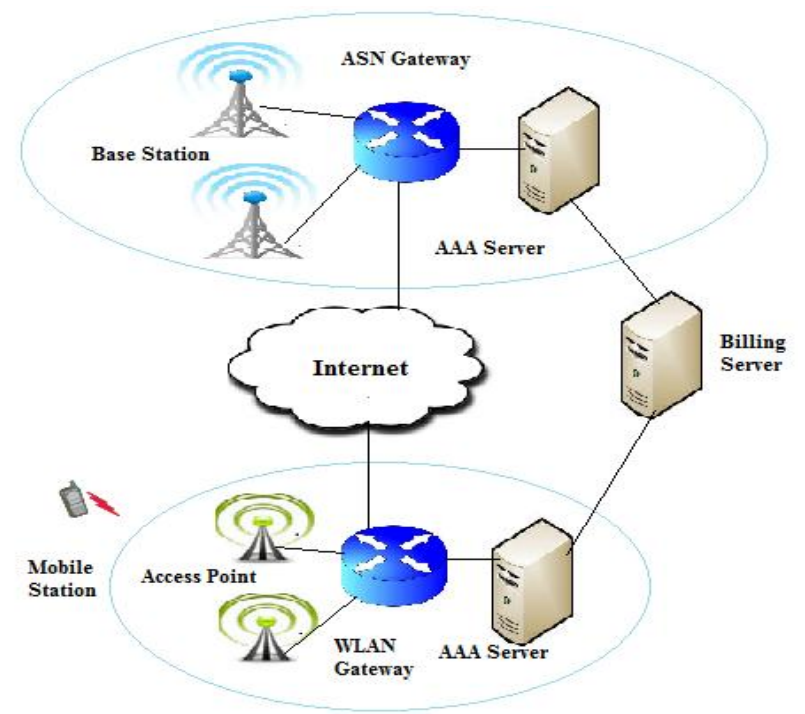

Fig- 4: Loosely coupled interworking of WLAN and WIMAX

(ii)Tight coupling: In tight coupling internetworking approach, WLAN network is connected directly to the WIMAX network. The tight coupling interworking can be done by connecting the WLAN network to WIMAX through WIMAX - Access Service Network gateway as mentioned in Fig-5. The advantage of using tight coupling interworking is, the efficiency achieved during mobility i.e., WIMAX mobility features are applied to those networks that are integrated with the WIMAX network. Therefore, seamless network mobility can be achieved in case of inter-domain handover. Also, packet loss gets reduced to a greater extent thereby minimizes the service degradation caused during handover to the new network.

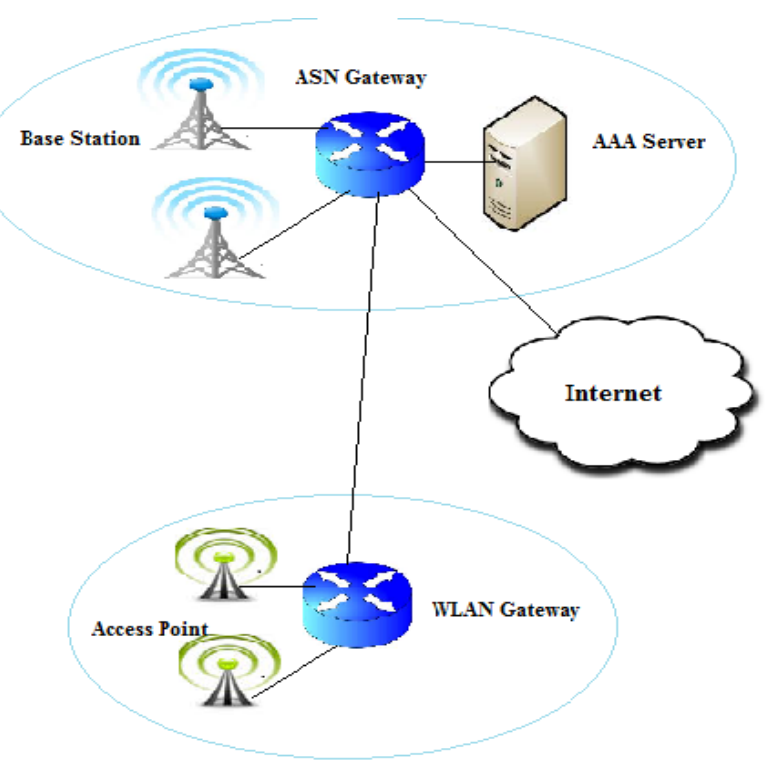

Fig- 5: Tightly coupled interworking of WLAN and WIMAX

\subsection{Mobile IP}

In Mobile IPv6, each Mobile Node (MN) is identified by means of a static IP address called home address. The MN can be reached by means of static home address when it moves to the foreign network. The permanent home address of the mobile users is maintained in the Home Agent database (HA). When the MN moves to a foreign network, it is allocated a new address called as Care of Address (CoA). The MN updates the CoA with the home network's HA and $\mathrm{CN}$ by means of binding update message. Since HA is updated with the CoA entry, it facilitates location management and routing of packets when $\mathrm{MN}$ is on the move. MIPv6 is a globalized IP mobility management protocol, where a MN maintain its connection with their home agent throughout the movement of MN from network to network. In order to acquire CoA from new network, the $\mathrm{MN}$ has to perform certain operations that causes delays. These include movement detection of MN, configuration of new CoA including Duplicate Address Detection (DAD) , and sending registration or binding update (BU) message to either HA or CN. The overall handoff latency in MIPv6 mechanism can be categorized as two types namely layer 2 (L2) and layer 3 (L3) delays. In L2 the total delays are sum of delay caused during scanning for available network, performing authentication to the new network and getting associated with the newer one. In L3 the total delays are sum of delays caused during movement detection, configuration of new CoA by new network including DAD delay, delay caused during Registration with HA and delay caused while sending binding update message to the HA/ CN. 


\subsection{Media Independent Handover}

IEEE 802.21 Media Independent Handover ensures interoperability between different types of wired access networks and wireless access networks. The purpose of IEEE 802.21 is to mainly improvise the quality of experience of the mobile devices when handover is done between IEEE 802 networks. Inspite of the difference in types of media involved both wired and wireless, IEEE 802.21 makes mobile devices to perform seamless IP handover. These mechanisms are also used to facilitate handover between IEEE 802 kind of networks and non IEEE 802 kind of networks. As in Fig 6. this standard defines MIHF services which promotes handovers between heterogeneous access network. MIHF is the heart of media independent handover service and comprises three functional services namely Media Independent Event Service (MIES), Media Independent Information Service (MIIS) and Media Independent Command Service (MICS).

Media Independent Event Service (MIES): This service detects the changes on both the physical and link layer. When there any changes in the link layer characteristics events are triggered by making use of event service. Events that are originated from MIHF are referred to as $\mathrm{MIH}$ Events and those events that are originated from any lower layer are referred to as Link Events. MIH events or Link events originates within the protocol stack of a $\mathrm{MN}$ or network. The destination of MIH event is either MIHF that is present in the remote node or any upper layer entity that is present within the same protocol stack. In case of local events, event messages are propagated from the lower physical or data link layer to the MIHF and from MIHF to any upper layer of the same protocol stack. In case of remote events, event messages are propagated from the MIHF present in one protocol stack to the MIHF present in the peer/remote protocol stack. One of the protocol stack is present in the MN while the other is present in a fixed network entity. The MIES triggers events such as events that are triggered during state change and they are Link Up, Link Down, Link_Going_Up, Link_Going_Down and Link parameter changes.

Media Independent Information Service (MIIS): The media independent information service (MIIS) defines a framework and mechanisms through which a MIHF could discover and obtain the required candidate network information existing within a particular geographical area to trigger the handover to the foreign network. The information is made available to both lower physical / link layer and higher network layer. MIIS provides parameters such as MAC address, channel information, data rate and security information of the foreign network. Information available about the higher layer services helps in taking more effective handover decision before the $\mathrm{MN}$ attaches to any foreign network.

Media Independent Command Service (MICS): This service enables higher layers to control the physical and data link layers to trigger the handover process. The higher layers are responsible for the reconfiguration of an appropriate network through a set of handover commands. When a MIHF receives a command, it executes those process that does the actual handover. Commands that are invoked by MIH users are referred to as MIH Commands and those commands that are invoked by MIHF are referred to as Link Commands. The destination of a command is either MIHF that is present within the same protocol stack or any lower layer that is present in the remote protocol stack In case of local commands, command messages are propagated from the MIH users to MIHF and then from MIHF to lower layers of the remote protocol stack. In case of remote commands, command messages are propagated from MIH users via MIHF present in one protocol stack to the MIHF present in a peer protocol stack. One of the protocol stack is present in the $\mathrm{MN}$ while the other is present in a fixed network entity.

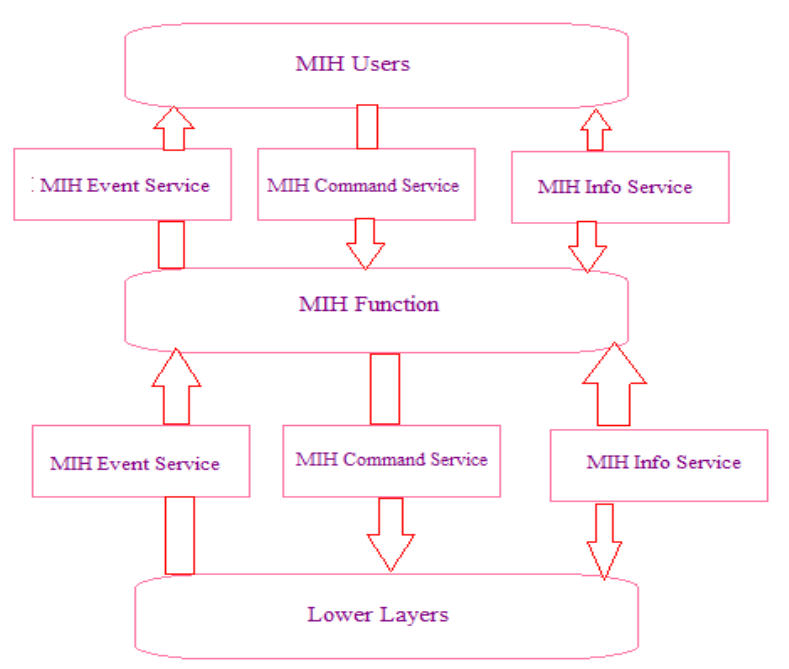

Fig-6: IEEE 802.21 MIH Architecture

\section{PROPOSED VERTICAL HANDOVER BETWEEN WLAN AND WIMAX}

Two different inter-networking environment namely loose coupling and tight coupling is created for WLAN/WIMAX networks and analysed the performance of handover delay. The first scenario describes the vertical handover for WLAN / WIMAX networks by considering RSS using IEEE 802.21 MIH for handover in both interworking architecture. The WIMAX base station provides maximum coverage area, whereas WLAN access point has less coverage area and hence initiates need for handover to the WIMAX network. Initially the mobile node is present in the WLAN network coverage area. The link layer periodically reports RSS value to the MIH user that is embedded in WLAN access point by making use of $\mathrm{MIH}$ event service command. If the LINK_GOING_DOWN event is triggered, MIH user initiate the necessity to do handover to the candidate network. The MIH user discovers the candidate network by sending MIH_Get_Information_Request to the information server. MIHU checks RSS of the WIMAX. Since signal strength value of the WIMAX network is greater than the threshold value configured in MN, it make sure that WIMAX network is currently accessible. At handover execution phase, MN 
send MIH_MN_HO_Commit_Req to the candidate network. After receiving MIH_MN_HO_Commit_Res handover to WIMAX network is initiated by making of MIH command service command. By means of experimentation it was analyzed that handover delay is maximum since the connection is not transferred completely to new PoA when handover is done based on RSS. Also handover due to RSS may lead to false handovers thereby again maximizing the handover delay.

Second scenario is the proposed vertical handover that is implemented based on IEEE 802.21 MIH standard where the handover is done by considering RSS and bandwidth of the neighboring network. This scenario is also implemented in both interworking architecture. Initially the mobile node is present in the WLAN network coverage area. The link layer periodically reports RSS measurement to the MIH user that is embedded in WLAN access point by making use of MIH event service command. If the LINK_GOING_DOWN event is triggered, MIH user initiate the need to do handover to the candidate network.

The MIH user discovers the candidate network by sending MIH_Get_Information_Request to the information server. MIHU checks RSS and bandwidth of the WIMAX. Since RSS and bandwidth of the WIMAX network is greater than the threshold value that is configured in $\mathrm{MN}$, it make sure that WIMAX network is currently accessible. At handover execution phase, MN send MIH_MN_HO_Commit_Req to the candidate network. After receiving MIH_MN_HO_Commit_Res from candidate network, handover to WIMAX network is initiated by making of MIH command service command. Here, the mobile node starts handoff to the WIMAX network within fraction of seconds and hence the delay is minimized.

\section{RESULTS AND DISCUSSIONS}

Table-1 shows the handover latency for the MIPv6 with/without using the MIH services. In MIPv6, using the MIH services the time taken for discovery of router is removed Handover latency is mainly due to time taken during duplicate address detection, link layer mechanism and binding to the candidate network.

Table-1: Handover latency for the MIPv6 with/without the MIH scheme.

\begin{tabular}{|l|l|}
\hline Handover mechanism & Handover Latency \\
\hline MIPv6 & $D_{\mathrm{L} 2}+D_{\mathrm{RD}}+\mathrm{D}_{\mathrm{DAD}}+\mathrm{D}_{\mathrm{BU}}$ \\
\hline MIPv6 + MIH (RSS) & $\mathrm{D}_{\mathrm{L} 2}+\mathrm{D}_{\mathrm{DAD}}+\mathrm{D}_{\mathrm{BU}}$ \\
\hline
\end{tabular}

In the first scenario ,for loose coupling architecture when MN is moving out of WLAN network coverage it measures RSS from candidate network. MN here identifies WIMAX network by means of information request/response messages and then joins the WIMAX network. So if we analyze WLAN and WIMAX load they are both balanced because WLAN traffic is shared to WIMAX network. As in Fig 8. it can be found that handover latency gets increased because the Mobile Node breaks the connection from WLAN network before it has moved completely to WIMAX network.

In the first scenario ,for tight coupling architecture when $\mathrm{MN}$ is moving out of WLAN network coverage it measures RSS from candidate network. MN here identifies WIMAX network by means of information request/response messages and then joins the WIMAX network. From Fig 7. it can be found that MN starts its scanning activity and received the response from WIMAX about its availability to MN. As in Fig 9. it can be found that handover delay is less when compared to loose coupling because Mobile Node receives traffic from both WLAN and WIMAX network until the Mobile Node has moved completely out of WLAN network.

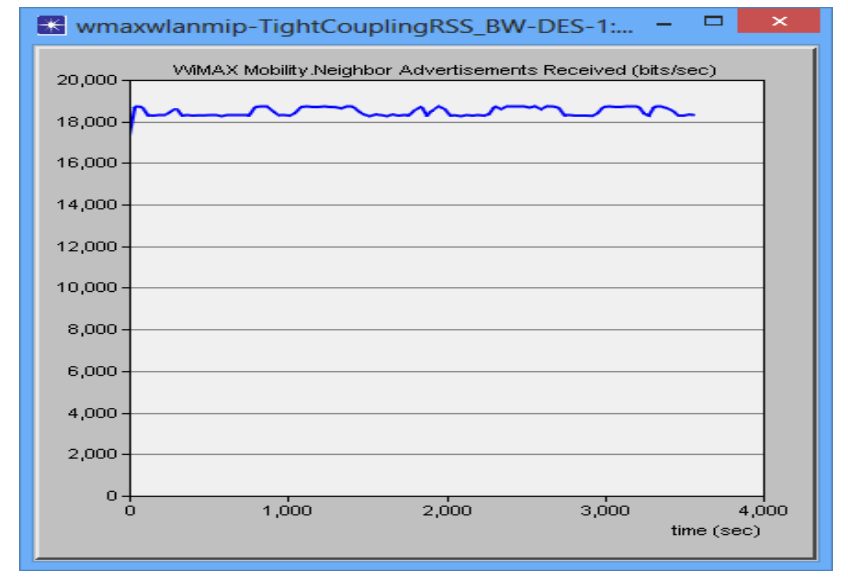

Fig-7: WIMAX Neighbor Advertisement Received

In the second scenario, for loose coupling the Mobile Node is present initially in WLAN network coverage and exchanges the data with correspondent node. If the link going down event is triggered, access point initiates the need to do handover to the candidate network. After receiving the response from the candidate network say WIMAX, it tries to get bandwidth of the candidate network. MIHU checks the RSS and bandwidth availability in WIMAX. If RSS and bandwidth of WIMAX is found to be greater than the threshold value, WiMAX is chosen as the target network for handover process. MIH command service is initiated to perform handover to WIMAX network.

The drawbacks with the existing scheme are that, the MN has to scan for available candidate network and then report the scanning result to MN i.e. RSS values for all detected candidate network and therefore it may result in high handover latency. Also there are lots of possibility of handover failures because if the handover was done to the new network but the network is unable to provide the services due to low bandwidth, then there is no use of handing over to the new network. From Fig -8, it can be seen that the proposed scheme serves to be advantageous when compared to the existing scheme where handover was done using the signal strength. 
In the second scenario, for tight coupling architecture the Mobile Node roams in WLAN network coverage area and exchanges the data with correspondent node. If the link going down event is triggered Access Point initiates the need to do handover to the candidate network. After receiving the response from the candidate network say WIMAX, it tries to get bandwidth of the candidate network. After checking the resource availability in WIMAX network, Mobile Node triggers handoff to the WIMAX network within fraction of seconds. From Fig -9.it is clearly depicted that handoff delay is less when compared to loose coupling because seamless mobility is achieved since WLAN traffic traverse via WIMAX network and also mobile node does handoff only when the resources like bandwidth is satisfied.

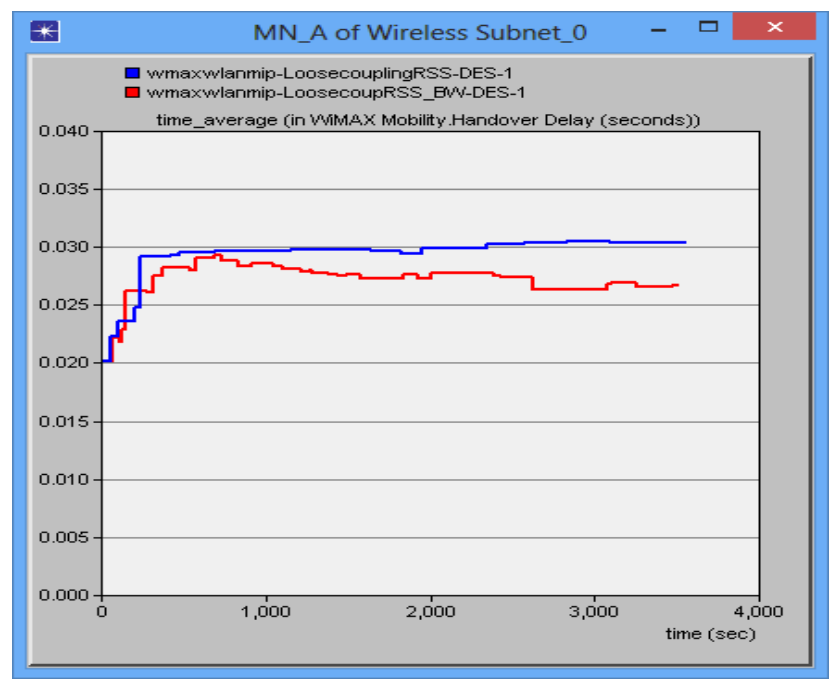

Fig -8: Handover Latency for Loose Coupling scenario

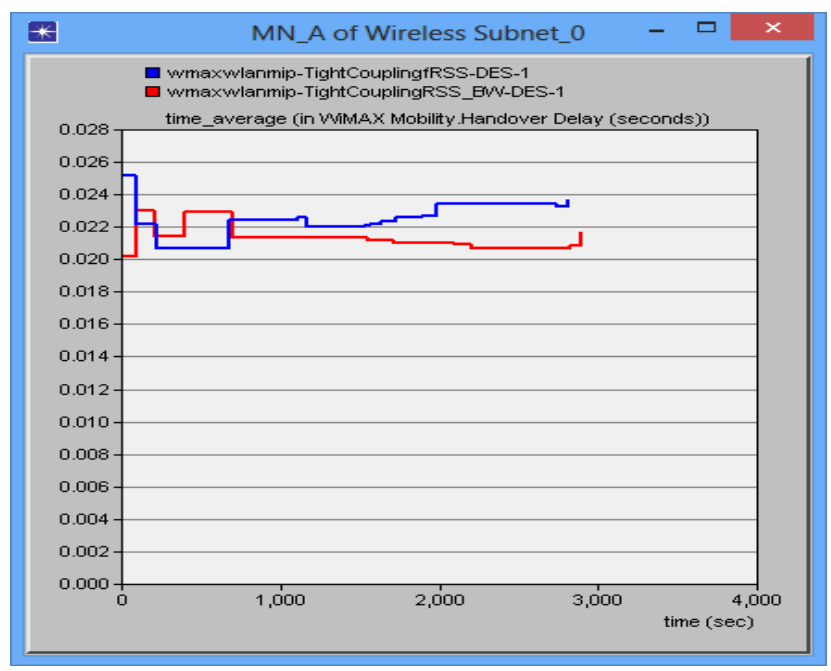

Fig -9: Handover Latency for Tight Coupling scenario

From Fig- 10. it is clearly depicted that the throughput of the loosely coupled and tightly coupled interworking of WLAN and WIMAX with RSS and bandwidth is high when compared to handover done using signal strength alone. On the whole, throughput of the loosely coupled architecture is very less when compared to the tightly coupled interworking architecture irrespective of the parameters considered for vertical handover. This is because in tightly coupled architecture data traffic of WLAN traverse via WIMAX network thus providing seamless connectivity and continuous traffic flow. From Fig -11 it is clearly depicted that load of WIMAX network is high in tightly coupled architecture when compared to loosely compared architecture because it can handle high traffic flow when compared to loosely coupled architecture.

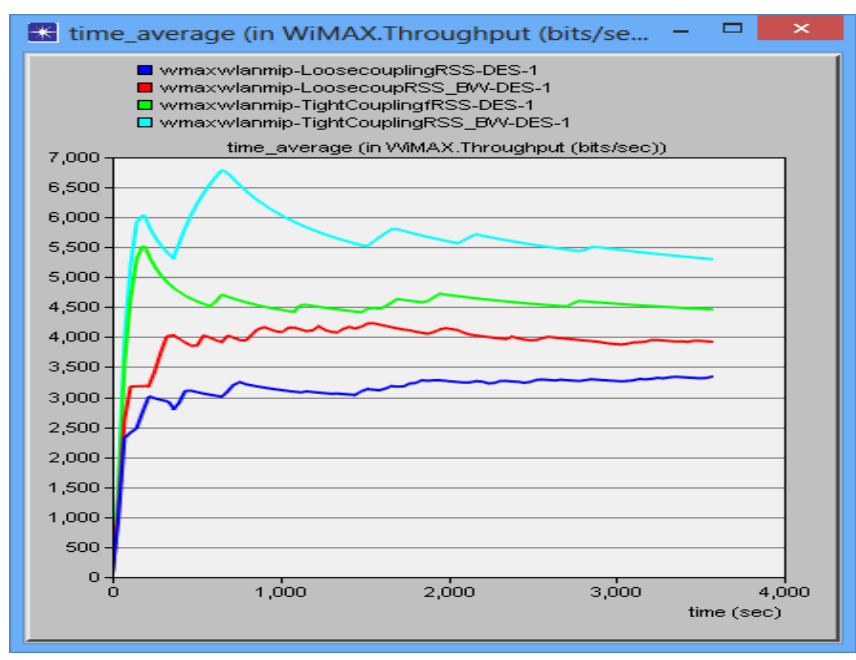

Fig -10: Throughput of Loose Coupling and Tight Coupling scenario

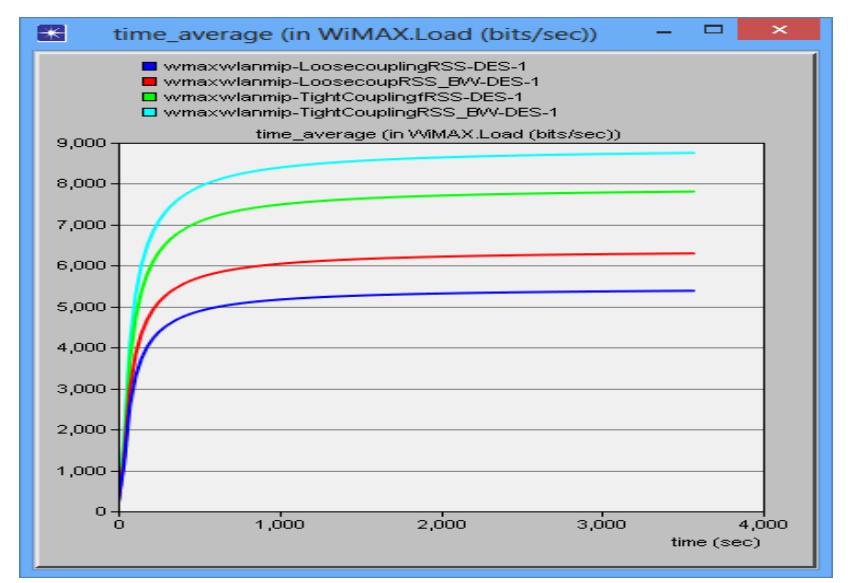

Fig -11: WIMAX Load for Loose Coupling and Tight Coupling scenario

\section{CONCLUSIONS}

The tightly coupled interworking of WLAN / WIMAX using signal strength and bandwidth with MIH provides relatively less handover delay when compared to handover done using signal strength alone. The same applies to loosely coupled interworking of WLAN / WIMAX. Also, it is shown that tightly coupled interworking of networks is very efficient when compared to loosely coupled network in both the scenarios. In tightly coupled interworking of WLAN/WIMAX, after handover is completed, the traffic flows through WIMAX network and load is balanced in 
both the networks. The resource allocated by WLAN to Mobile Node is released thus providing seamless connectivity whereas in the loosely coupled interworking of networks, there is an interruption in service while Mobile Node moves from WLAN to WIMAX thereby increasing handover latency. Future work aims at application of different mobility management protocols at different layers and analyze those factors that are required for efficient vertical handover from WLAN to WIMAX.

\section{REFERENCES}

[1] Stenio Fernandes and Ahmed Karmouch ,"Vertical Mobility Management Architectures in Wireless Networks A Comprehensive Survey and Future Directions", IEEE Communications Surveys , Vol. 14, 2012.

[2] Ms.Farah M. Khan ,Prof.Satish K. Shah ,Ms.Dharmishtha D. Vishwakarma , "A Review on Media Independent Handover Services for Heterogeneous Wireless Communication Networks", International Journal of Electronics and Computer Science Engineering, IJECSE Volume 1, Number 3 , 2012.

[3] Son Tran-Trong, Shahnaza Tursunova, and YoungTak Kim , "Enhanced Vertical Handover in Mobile IPV6 with Media Independent Handover Services and Advance Duplicate Address Detection", International Journal of computer applications, Volume 2,August 2010.

[4] Abhishek Dhiman and Karamjit Singh Sandha , "Vertical and Horizontal Handover in Heterogeneous Wireless Networks using OPNET", International Journal of Engineering Research \& Technology (IJERT), Vol. 2 Issue 6, June 2013.

[5] Safdar Rizvi, Asif Aziz, N.M. Saad, Nasrullah Armi, and Mohd Zuki Yusoff , " Tight Coupling Internetworking Between UMTS and WLAN Challenges, Design Architectures and Simulation Analysis "International Journal of Computer Networks (IJCN), Volume (3) Issue (2) 2011.

[6] M. M. A. Khan, Omar Arafat, K. Dimyati and Fatima Seeme, "Seamless Mobility Management between IP-based Networks", International Journal of Networks and Communications, 2012.

[7] Jong-Tae Park, Seung-Mu Lee, and Seung-Man Chun ,"P2P-based Mobility Management Protocol for Global Seamless Handover in Heterogeneous Wireless Networks", International Journal of Electronics and Computer Science Engineering, IJECSE Volume 1,2011.

[8] Wei wu, Alcatel, Nilanjan Banerjee, Kalyanbasu and Sajal k.Das, "SIP-based vertical handoff between WWANs and WLANs", IEEE Wireless Communications, June 2005.

[9] Ahmad H. Talaat ,Nael A. Hussein ,Hussein A. Elsayed and Hadia Elhennawy,"Real-time Traffic Performance for WiFi Handovers over MIPv4 versus MIPv6", International Journal of Computer Applications (0975 - 8887),Volume 26- No.8, July 2011.
[10] Dheyaa Jasim Kadhim ,Sanaa Shaker Abed , "Performance And Handoff Evaluation Of Heterogeneous Wireless Networks (Hwns) Using Opnet Simulator", International Journal of Electronics and Communication Engineering \& Technology (IJECET), Volume 4, Issue 2, March April, 2013, pp. 477-496.

[11] Kenichi Taniuchi, Yoshihiro Ohba and Victor Fajardo, Subir Das, Miriam Tauil, Yuu-Heng Cheng, Ashutosh Dutta, Donald Baker, Maya Yajnik, and David Famolari, "IEEE 802.21 Media Independent Handover Features, Applicability, and Realization",IEEE Communications Magazine ,January 2009.

[12] Ammar A. Bathich, Mohd Dani Baba, Muhammad Ibrahim," IEEE 802.21 Based Vertical Handover in WiFi and WiMAX Networks", IEEE Symposium on Computers \& Informatics, 2012.

[13] Meriem Kassar , Brigitte Kervella, Guy Pujolle , “An overview of vertical handover decision strategies in heterogeneous wireless networks ", Elsevier, Computer Communications 31 (2008).

\section{BIOGRAPHIES}

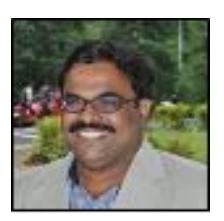

Dr. G. Zayaraz is currently working as a Professor in the Department of Computer Science and Engineering, Pondicherry Engineering College, Puducherry,India. Email:zayaraz@pec.edu

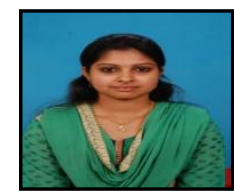

J. Kanchana Devi is currently pursuing Master's Degree program in Computer Science and Engineering in Pondicherry Engineering College, Puducherry, India. Email: kanchana88@gmail.com

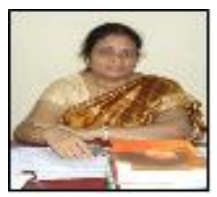

Dr. V. Vijayalakshmi is currently working as a Assistant Professor in the Department of Electronics Communication and Engineering, Pondicherry Engineering College, Puducherry, India.

Email: vivijizai@pec.edu

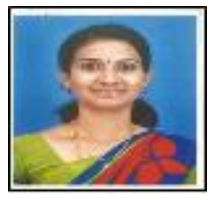

Mrs. V. Hemamalini is currently pursuing $\mathrm{PhD}$ in the area of Wireless security in Pondicherry Engineering College, Puducherry, India.

Email:cse.malini@gmail.com 